\title{
Estrategias didácticas para el fortalecimiento del proceso lector a nivel andragógico
}

\section{Didactic strategies for strengthening the reading process at the andragogic level}

\author{
Derling Mendoza V. \\ Universidad Iberoamericana del Ecuador-UNIB.E, Ecuador \\ Autor para correspondencia: dmendoza@unibe.edu.ec \\ Fecha de recepción: 10 de Octubre 2017 - Fecha de aceptación: 15 de Febrero de 2018
}

Resumen: La adquisición y desarrollo del proceso de lectura constituye para las instituciones Universitarias un desafío en la formación de lectores competentes, personas que adquieran habilidades para el disfrute de los textos, como una actividad voluntaria, donde no sólo se puede extraer la información, sino interpretarla, disfrutarla y vivirla para su formación académica. Por consiguiente, es elemental asumir posturas, para practicar la lectura dentro y fuera de la Universidad, donde se haga hincapié que el lector busque información, se entretenga, resuelva un problema práctico y pueda conocer otros mundos posibles. En este sentido, se desarrolló un estudio bajo la investigación de campo de carácter documental y dentro de la modalidad de proyecto factible, enmarcada en tres fases: diagnóstico, factibilidad, diseño de la propuesta. Por tanto, la población se conformó por (10) docentes y (25) estudiantes(as) de la Universidad Iberoamericana del Ecuador. Para efectos de recopilar la información se utilizó un cuestionario con varias alternativas, el cual fue sometido a un proceso de validez de contenido y confiabilidad utilizando el coeficiente de Alfa de Cronbach, obteniendo como resultado 0,81 el cual se considera confiable. A tal efecto, los resultados de la investigación permitieron fortificar la lectura a nivel andrológico, como también facilitar la orientación de los estudiantes e investigadores, para tomar posturas actitudinales de lectores competentes, autónomos y críticos en cada situación de la lectura.

Palabras Clave: estrategias didácticas; fortalecimiento; proceso lector

Abstract: The acquisition and development of the reading process constitutes for University institutions a challenge in the formation of competent readers, people who acquire skills for the enjoyment of the texts, as a voluntary activity, where not only the information can be extracted but interpreted, enjoy it and live it for your academic training. Therefore, it is fundamental to assume positions, to practice reading inside and outside the University, where it is emphasized that the reader seeks information, is entertained, solves a practical problem and can know other possible worlds. In this sense, a study was developed under field research of documentary nature and within the feasible project modality, framed in three phases: diagnosis, feasibility, proposal design. Therefore, the population was confirmed by (10) teacher and (25) students (as) of the Universidad Iberoamericana del Ecuador. In order to compile the information, a questionnaire with several alternatives was used, which was submitted to a content validity and reliability process using the Cronbach's alpha coefficient, obtaining a result of 0.81 which is considered reliable. To this end, the results of the research made it possible to fortify reading at the andrological level, as well as to facilitate the orientation of students and researchers, to take attitudes of competent, autonomous and critical readers in each situation of reading.

Key Words: didactic strategies; strengthening; reading process 


\section{Introducción}

Al transcurrir del tiempo el hombre ha creado diferentes formas de comunicación, la lectura forma parte de esta, ha sido un instrumento trascendental para la escolarización universitaria, socialización, el desarrollo intelectual y profesionalización del ser humano. No obstante, aprenderla requiere directamente la capacidad de comprensión lectora, es decir; convertir las palabras en significados, esta construcción de significados debe partir de la interacción entre el texto, el contexto y el lector, estos elementos juntos determinan la comprensión del mismo, según (Pérez, 1999, pp. 21):

Hoy sabemos que toda la lectura y la escritura es un diálogo entre el texto, contexto y el lector, que el significado no se descubre sino se construye y que, por eso, es posible leer de lo que uno ya sabe.

Por otra parte, (Gispert, 2000, pp. 92), plantea que; “en la actualidad, el proceso perceptivo se identifica con la actividad que permite al lector reconocer el texto escrito como representación del lenguaje hablado". Es decir, como el proceso permite encontrar el significado de las palabras. Estos dos autores están de acuerdo que la lectura es más que la decodificación del texto, el lector debe construir el significado desde la experiencia previa de su conocimiento entrelazándolo con la nueva información, para acceder a un conocimiento amplio y actualizado, igualmente para (Dorronzoro, 2004, pp. 8):

En nuestro caso, en el contexto universitario la principal finalidad asignada a la lectura y la escritura es la construcción, apropiación del conocimiento dentro de un determinado campo de saber. (..) Esta meta de la lectura es designada por la institución, entre otras cosas, por medio de las producciones verbales efectivas propias del contexto, es decir, los textos orales y escritos, relativos a los diferentes campos del saber, que circulan en el medio académico y que organizan de una manera particular los conocimientos de los miembros de una misma formación social. En este sentido, creemos que <a través de la enseñanza> se comunican, se solicitan y se permiten determinados usos de la lengua escrita, asignando de esta forma determinados propósitos a la lectura.

A nivel universitario, la comunidad académica y los investigadores conllevan un contexto diferente al inculcado previamente en la etapa del bachillerato, donde al estudiante se le recuerda de forma frecuente y constante su aplicabilidad, en la educación superior cuyas características específicas inciden en sus formas de actuación discursiva, los estudiantes conforman sus conocimientos, creencias y acciones - muchas veces en un status implícito en el que pocas ocasiones requieren a la búsqueda de información por la lectura. Los compendios del entorno institucional deben considerarse como atributos consecuentes que determinan las disertaciones y las prácticas discursivas de quienes participan en la interacción lectora.

De igual manera, entre alguna de las causas que genera esta situación obedece a una práctica rutinaria, poco incentivo hacia el proceso lector, ausencia de círculos de lectura, poca utilización de estrategias didácticas que ayuden a formar lectores eficaces y competentes. Por otra parte, el desarrollo de la lectura no está basada dentro de procesos significativos que 
incentiven a los estudiantes(as) a identificarse y disfrutar de ellos. Aunado a esto, se suma la falta de interés de la sociedad investigativa en concebir la lectura como una herramienta valiosa que les permite superar con menor dificultad los procesos de aprendizaje y estudio. En este orden de ideas, se observó que, en la comunidad estudiantil, existen debilidades en el proceso lector, esto se evidencia en la realización del diagnóstico ejecutado a los estudiantes(as) que ingresan a la Universidad, lo que puede conllevar a bajos promedios en las diferentes asignaturas.

De igual manera, se evidenció que no se desarrollan planes de nivelación educacional desde el bachillerato para fortalecer habilidades en los estudiantes que ingresan buscando consolidar competencias sobre la lectura. Asimismo, no se crean grupos de trabajos entre los docentes para intercambiar experiencias en la búsqueda de estrategias didácticas que proyecten la lectura para hacer de los estudiantes(as) lectores, donde centre su enseñanza en la formación de seres que sean capaces de comprender los diversos textos escritos, es decir, un docente que enseñe a leer, porque le gusta la lectura, que se exprese con adecuación para ampliar el vocabulario científico del alumno(a), que enseñe procesos y actitudes, no sólo conceptos y desarrolle en ellos unas competencias que les permita comunicarse con propiedades en la comunidad investiga donde cursan sus estudios.

De no ser así, trae como consecuencia el aislamiento entre el texto, contexto y el lector y la esencia de contrición del significado, seguirán manteniendo el poco interés de los estudiantes(as) hacia el proceso lector, continuando la enseñanza de la lectura como una simple decodificación del texto. Por ello, la necesidad de incentivar al estudiante universitario a utilizar diferentes espacios y estrategias didácticas para desarrollar competencias lectoras necesarias para cambiar los esquemas investigativos que les permita el desarrollo pleno de su intelecto.

En base a estas consideraciones, la lectura debe asumirse como un acto donde el lector se relaciona activamente con el texto, en un diálogo en el que se activan varias destrezas de pensamientos y expresión. De allí, que la acción del docente es decisiva para permitir el disfrute y generar interés en los estudiantes por lo que va a leer al inicio del semestre de estudios. De igual manera, debe existir un carácter motivacional para desarrollar en las aulas situaciones de lecturas auténticas, donde los alumnos lean por su propia voluntad y generen acercamientos positivos para encaminar el proceso lector.

Por otra parte, la lectura debe tener sentido y poseer la cualidad del carácter significativo, para que el investigador pueda comprender y lo relacione a su conocimiento e interés. De allí, que la comprensión se vincula entonces estrechamente con la visión que cada uno tiene del texto, no se puede pretender una interpretación única y objetiva. Por tanto, el aprendizaje de la lectura se debe realizar bajo condiciones significativas, es decir, incentivándolos al gusto por leer.

Ante la presente connotación, el estudio realizado tuvo como objetivo proponer estrategias didácticas para el fortalecimiento del proceso lector en los estudiantes de la Universidad Iberoamericana del Ecuador, para incentivar el proceso investigativo, asumiendo que el lenguaje es el vehículo por el cual se transmiten pensamientos y permite al ser humano satisfacer la necesidad de comunicarse con los demás. Por tanto, se recomienda el uso de estrategias didácticas que permitan a los estudiantes descubrir y disfrutar de la lectoescritura, generando posibilidades de éxitos en el proceso de aprendizaje. Asimismo, desenvolverse dentro 
del contexto sociocultural y mantenerse informados de todo lo que acontece dentro del ambiente donde se desenvuelve y fuera de él.

\section{Metodología}

En correspondencia con el estudio, se utilizó la investigación de campo, que de acuerdo a (Hernández, Fernández y Baptista, 2003, pp. 114) puede definirse como: "Aquel que se realiza mediante la recolección de los datos directamente de la realidad o del lugar donde se efectuará el estudio mediante la aplicación de técnicas de encuestas, entrevistas y observación directa". En este sentido, se entiende por investigación de campo el análisis sistemático de problemas en la realidad, donde los mismos serán recogidos en forma directa. Del mismo modo, se ubicó en una investigación documental, definida por (Fidias, 2012, pp. 27), como "un proceso basado en la búsqueda, recuperación, análisis, crítica e interpretación de datos secundarios, es decir, los obtenidos y registrados por otros investigadores en fuentes documentales: impresas, audiovisuales o electrónicas". Como en toda investigación, el propósito de este diseño fue fortalecer la elaboración de la propuesta.

En este orden de ideas, se concibe la investigación dentro de la modalidad de investigación proyectiva la cual permite solucionar necesidades de una institución y proponer alternativas de cambio. En este sentido, (Hurtado, 2000, pp. 325) señala:

La investigación proyectiva consiste en la elaboración de una propuesta o de un modelo como solución a un problema o una necesidad de tipo práctico ya sea de un grupo social o una institución en un área particular del conocimiento a partir de un diagnóstico preciso de las necesidades del momento, los procesos explicativos o generadores involucrados y las tendencias futuras.

Según los criterios expresados por la autora, la elaboración de una propuesta o de un modelo, constituye la solución a un problema, de esta manera se visualiza la relación directa con la Universidad, la cual permitió facilitar los datos necesarios para el desarrollo de la investigación, como también la articulación de la documentación bibliográfica que respalda el basamento teórico del estudio.

Para el desarrollo de esta modalidad se consideraron tres fases que (Hernández, 2014, pp. 11), las describe de la siguiente manera, "Fase I: diagnostico, Fase II: proceso metodológico, y fase III: diseño de la propuesta". En base a las fases descritas por la autora el investigador realizo proyecta la investigación de la siguiente manera:

Fase I: Diagnóstico: Constituye la parte descriptiva del proceso, allí se trata de hacer una descripción detallada de la situación que se desea mejorar, es describir lo más objetivamente posible la realidad que de acuerdo a la perspectiva del investigador presenta serias debilidades, $\mathrm{y}$, por lo tanto, se hace necesario proponer un proyecto de acción para mejorarla.

Se estudió la situación evidenciada a fin de ratificar los hallazgos que evidencian las debilidades sobre la lectura, para efectos de recopilar la información y elaborar el diagnóstico se utilizó como técnica la entrevista acompañada de un cuestionario de varias alternativas. Luego de 
confrontadas las opiniones y relacionadas la similitud de las respuestas, se conformaron las actividades que van a transformar la acción ejecutada por los docentes de la Universidad Iberoamericana del Ecuador, en referencia al proceso lector. Buscando ubicar una serie de estrategias que concebidas dentro de espacios significativos incentivarán a los estudiantes(as) a identificarse y valorar la importancia de la lectura para su desarrollo investigativo.

Fase II: Corresponde a la segunda fase del proceso metodológico de la modalidad, donde se establecen los criterios que permiten asegurar el uso óptimo de los recursos empleados, así como los efectos del proyecto en el área o sector al que se destina. Durante el proceso de factibilidad, se establecieron los criterios que permitieron asegurar el uso óptimo de los recursos utilizados, así como los efectos del proyecto en el área investigativa de la Universidad Iberoamericana del Ecuador (UNIB.E). En la presente fase se estableció la viabilidad a la propuesta, enfocada en los recursos humanos, económicos e institucionales. Asimismo, se elaboró un cronograma de trabajo para un mayor desenvolvimiento en las actividades a ejecutarse. Las mismas, se relacionaron con todo lo concerniente a la Universidad y al contexto investigativo, a fin de que la propuesta no presentara limitantes.

Fase III: Diseño de la Propuesta: Representando la tercera fase identificada en el esquema general, es definida por la autora, como la fase en la cual se define el proyecto con fundamento en los resultados del diagnóstico. Es en esta fase donde se diseña la propuesta de solución a las necesidades, con especificación del modelo, objetivos, metas, procesos técnicos, actividades, recursos y calendarización.

La misma autora descrita anteriormente señala la importancia de la fase III que radica, en que se combinan los recursos humanos, técnicos y financieros, poniendo en juego el máximo de creatividad, para la obtención de ciertos logros en la solución de una problemática en un contexto y tiempo determinado.

El diseño del proyecto de estudio fue producto de un proceso de planificación, donde el investigador ralentizo las actividades de campo y para inducir en la preparación del modelo, el cual se formó partir del diagnóstico previamente realizado. En esta fase, se planificaron todas las actividades a ejecutarse, los acuerdos y compromisos asumir, para llegar a logros satisfactorios. Todo ello tiene su fuente en la vivencia a partir de los aportes de los colaboradores y el trabajo del investigador para dar respuesta al objetivo planteado de diseñar estrategias didácticas para el fortalecimiento del proceso lector a nivel andragógico en la Universidad Iberoamericana del Ecuador.

\section{Población del estudio}

Se entiende como población según (Arias, 2006, pp. 81), "Es un conjunto finito o infinito de elementos con características comunes para los cuales serán extensivas las conclusiones de la investigación. Está queda delimitada por problema y por los objetivos del estudio". Para efectos del estudio, la población estuvo conformada por diez (10) docentes de distintas áreas y veinticinco (25) estudiantes de ellos, (5) participantes de Ingeniería de Software, (5) de Hospitalidad, Hotelería y Turismo, (5) de Gastronomía, (5) de Producción para medios de 
comunicación y (5) estudiantes de Nutrición y Dietética, que conforman las carreras que ofrece la UNIB.E, ubicada en la Ciudad de Quito, Ecuador.

Validez y confiabilidad del instrumento

\section{Validez}

Según los autores (Prieto y Delgado, 2010, pp. 71) apuntan que "la validez se refiere al grado en que la evidencia empírica y la teoría apoyan la interpretación de las puntuaciones de los tests relacionada con un uso específico".

Existen varios tipos de validez. A tal efecto, para los procesos de la investigación en estudio, en referencia a los instrumentos aplicados, se utilizó la validez de contenido que a través del juicio de experto se puede constatar la pertinencia del instrumento con los objetivos que se persiguen. A tal efecto, (Chiner, 2008, pp. 2) establece que "la validez de contenido se utiliza principalmente con tests de rendimiento, y especialmente con los tests educativos y tests referidos al criterio. En este tipo de tests se trata de comprobar los conocimientos respecto a una materia o un curso". Por tanto, se pidió la colaboración a tres (03) docentes anexos a la Universidad objeto de estudio.

\section{Confiabilidad}

Para (Bernal, 2006, pp. 214), "la confiabilidad de un cuestionario se refiere a la consistencia de las puntuaciones obtenidas por las mismas personas, cuando se las examina en distintas ocasiones con los mismos cuestionarios". En este sentido, se aplicó una prueba donde se seleccionó un grupo diferente a la muestra de estudio, pero que representan características simultáneas y que pueden generar procesos para medir la confiabilidad. Asimismo, se utilizó el coeficiente de Alfa de Cronbach dentro de la siguiente formula:

$$
\alpha=\frac{K}{K-1} \cdot\left[1-\frac{\Sigma S i^{2}}{S}\right]
$$

$\mathrm{K}=$ Número de Ítems

$\mathrm{Si}^{2}=$ Varianza de los Números de cada Ítem. $\mathrm{S}=$ Varianza de los Puntajes Torales.

$$
\alpha=\frac{11}{11-1} \cdot\left[1-\frac{10.40}{40}\right]
$$

$\alpha=0.81$

Es el coeficiente de confiabilidad. Resultado que expresa una alta confiabilidad. 


\section{Resultados y discusión}

De acuerdo a los resultados se logró asumir que los estudiantes muestra del estudio que ejecutan sus estudios en diferentes carreras de la Universidad, no tienen presente que la lectura además de un hábito es una actividad intelectual que se manifiesta claramente al interpretar el texto y reconstruir su significado, por ello, es conveniente ubicar estrategias didácticas, espacios apropiados, selección de obras, para fortalecer el gusto hacia la lectura para resultar una actividad intelectual, investigativa, formativa y placentera; jamás debe ser tomada como un hecho obligado o impuesto.

Por otra parte, los alumnos no demostraron poseer orientación de los procesos básicos de la lectura, para ofrecer amplias oportunidades donde los estudiantes practiquen la lectura, pero como una necesidad atractiva. De igual manera mediante el diagnostico, se evidencio que no se presentaron habilidades y destrezas de los estudiantes hacia la lectura.

Donde (Ynclán, 1997, pp. 263) expresa que:

... aprender a leer supone no sólo el aprendizaje y automatización, sino fundamentalmente el aprendizaje de diversas estrategias que facilitan la combinación de la información del texto y la que procede de los conocimientos del lector, para construir la representación del significado global del texto. Por consiguiente la práctica docente respecto de la lectura y las habilidades de comprensión deberían experimentar cambios significativos en cuanto al qué y cómo enseñar.

En este orden de ideas, y según la autora se evidenció también que el poco uso de estrategias didácticas no conlleva a incentivar a los estudiantes hacia el proceso lector, ya que los mismos tienen un sistema de aprendizaje academicista desde su formación en el bachillerato, en el cual, existe el predominio del docente como único portador de la información para la adquisición del conocimiento, transmitiéndose de carácter unidireccional.

Asimismo, que les permita concebir la lectura como una actividad significativa en un rango científico y que fomente mayor acceso hacia el aprendizaje significativo. De igual manera, es necesario incentivar al estudiantado hacia el deseo por la lectura, pero satisfaciendo sus necesidades e intereses por su formación académica. Además, que puedan tener la oportunidad de seleccionar las lecturas que más les llame la atención.

\section{Conclusiones y recomendaciones}

La educación, es un proceso integral e interrumpido que acompaña al individuo desde su nacimiento, contribuyendo a que el cambio de conducta sea positivo en la medida que permita desarrollar habilidades y destrezas para dar seguridad, asimismo, al proporcionar una información para formular juicios de valores en que apoyarse, actuar, investigar, analizar, expresar, criticar y capacitado para participar en el medio social donde se desenvuelve. 
Bajo esta perspectiva, el sistema de educación superior del Ecuador posee la esencia pluralista, dando apertura al pensamiento y desarrollo científico para la formación de un individuo que contribuya al desarrollo de la república, donde La Universidad Iberoamericana del Ecuador le corresponde producir propuestas y planteamientos para buscar la solución de problemas, en este sentido, de carácter formativo del estudiantado a nivel andragógico, profesional, técnico y científico para que el individuo aporte a la sociedad todos los aspectos un ser justo, solidario e innovador.

Del mismo modo, para rectificar la importancia de la lectura, basta con pensar en el número de veces que, en un día normal, se debe leer para obtener información y orientación en el medio. Es decir, desde el cartel en la calle que indica una dirección pasando por el periódico para estar informado de los últimos acontecimientos o el manual de desempeño laboral que ayuda al desarrollo del trabajo cotidiano, hasta llegar a las obras literarias, artículos científicos, que permiten la recreación y la ampliación de experiencias.

En el contexto universitario, (Dorronzoro, 2004, pp. 8), expresa que:

...la principal finalidad asignada a la lectura y la escritura es la construcción, apropiación del conocimiento dentro de un determinado campo de saber. Esta meta de la lectura es designada por la institución, entre otras cosas, por medio de las producciones verbales efectivas propias del contexto, es decir, los textos orales y escritos, relativos a los diferentes campos del saber, que circulan en el medio académico y que organizan de una manera particular los conocimientos de los miembros de una misma formación social. En este sentido, creemos que a través de la enseñanza se comunican, se solicitan y se permiten determinados usos de la lengua escrita.

En la UNIB.E se destaca la necesidad de desarrollar destrezas de lectura, creación de hábitos, para lo cual hay sugerido la utilización de cambios metodológicos y procesos didácticos para lograr objetivos en el área de lengua conjuntamente con las áreas de otras carreras. Desde este punto de vista, las estrategias didácticas tienen como objetivo primordial caracterizar y globalizar los procesos de la lectura dentro de un abordaje que permita sustentar un aprendizaje de acuerdo a la información previa del alumno(a) para abrir procesos de aprendizajes relacionados con la internalización de la lectura.

La lectura como puerta de entrada a la cultura investigativa y académica, determina el factor de identidad, como vía para la instrucción ideológica universitaria. Por tanto, las estrategias didácticas propician situaciones comunicativas y dentro de ellas se pueden desatacar la elaboración de murales, periódicos, registros analógicos, ejercicios, animaciones las cuales permitirán que el proceso de la lectura se dinamice y sea significativo dentro y fuera de la UNIB.E. De allí, que los docentes deben tener presentes que la lectura está basada en el desarrollo de un conjunto de conductas que deliberadamente permiten la obtención de significado a partir de un material escrito.

De igual manera, los diligentes de esta temática han demostrado que la adquisición de habilidades de lectura permite un mejor dominio de la lengua. Por lo tanto, las estrategias 
didácticas fortalecerán el proceso de la lectura en la UNIB.E objeto de estudio permitiéndole a los docentes proyectar su práctica andragógica y a los estudiantes superar las debilidades en cuanto a este aspecto para constituirse en lectores potenciales.

\section{Factibilidad de la propuesta}

La factibilidad de la propuesta, desde el punto de vista investigativo (Dubs de Moya, 2002, pp. 15), lo enfatiza como "la posibilidad real de ejecución de la propuesta, en términos del grado de disponibilidad de recursos humanos, infraestructura, económicos, materiales, equipos y otros, necesarios para su funcionamiento". En la ejecución de la misma, es factible porque su acción orienta la necesidad de brindar acompañamiento a los docentes y a los estudiantes para el fortalecimiento de la lectura como un hecho significativo en el desarrollo investigativo del participante. En este sentido, para determinar la factibilidad de la propuesta se discutió con los docentes involucrados la inquietud del investigador, debido a la observación en los diferentes grados de las dificultades que presentan los alumnos ante el proceso lector.

En este orden de ideas, se discutió a su vez la importancia que supone el uso de estrategias didácticas como apoyo a este proceso. Asimismo, los colaboradores fueron participe en la elaboración de la propuesta por lo que queda definido que la misma será factible y sus actividades se desarrollarán a cabalidad, porque todos están interesados en contribuir a solucionar la problemática que viven los estudiantes que conforman la Universidad Iberoamericana del Ecuador.

Para su factibilidad, se seleccionó solamente a los estudiantes previamente descritos en la población y docentes de las distintas especialidades. Asimismo, las actividades planificadas pueden estar sujetas a cambios, debido a que la finalidad está basada en el máximo cumplimiento de las estrategias propuestas

\section{Factibilidad institucional}

En este sentido, quedo demostrado que la propuesta beneficia la calidad investigativa que debe desarrollar la UNIB.E puesto que fomentar la lectura y la capacidad de disfrutar de ella es un paso que provoca la motivación hacia la construcción del conocimiento.

\section{Factibilidad económica}

Dentro de este proceso no se presentó ninguna limitante, debido a que la propuesta no requiere de gastos exagerados y además que la UNIB.E ofrece como recurso primordial la Biblioteca, anexando de igual forma la biblioteca digital de la cual dispone se disponen los elibro y e-books tipo 7-24, estos se les conoce por la (Asociacion de Editores de Madrid, 2016, pp. 3) como:

...libro digital o libro electrónico o Ebook, formalmente el libro digital es cualquier texto almacenado en formato digital. Como tal, para leer esos libros son necesarios los programas denominados denominados lectores lectores, que 
pueden estar integrados integrados en los ordenadores ordenadores, teléfonos móviles o lo más reciente, lectores específicos basados en la tinta electrónica.

Factibilidad socio cultural

La misma se orienta en proyectar las inquietudes de la Constitución de La República del Ecuador quien establece en su artículo 27 que:

La educación se centrará en el ser humano y garantizará su desarrollo holístico, en el marco del respecto a los derechos humanos, al medio ambiente sustentable y a la democracia; será participativa, obligatoria, intercultural, democrática, incluyente y diversa, de calidad y calidez; impulsará la equidad de género, la justicia, la solidaridad y la paz; estimulará el sentido crítico, el arte y la cultura física, la iniciativa individual y comunitaria, y el desarrollo de competencias y capacidades para crear y trabajar.

De igual forma la factibilidad de la investigación se sustenta en base al (Plan Nacional Para el Buen Vivir, 2013-2017), donde su aplicación respalda:

La Revolución Cultural, que genera nuevos conceptos y valores ciudadanos para constituir una sociedad crítica, emprendedora, creativa y solidaria. Se vuelven fundamentales las industrias culturales y la democratización de la palabra. También implica una revolución educativa para formar ciudadanos con otra mentalidad, con valores asentados, con ética y con autoestima. El Buen Vivir significa, sobre todo, tener en el país una población con una gran dosis de autoestima y de confianza colectiva.

En la ejecución de la misma el proceso lector provee la formación crítica, cultural, científica e investigativa de los estudiantes. Por tanto, se deben buscar estrategias didácticas que incentiven a los alumnos a cada día concebir la lectura como un medio que los acerca a la información, las cuales se encuentran estructuradas en la siguiente propuesta. 


\section{Bibliografía}

Arias, F. (2006). El proyecto de investigación. Introducción a la metodología científica. Caracas: Episteme.

Asociacion de Editores de Madrid. (2016). Edición de libros digitales. Editorial Gadir. España. Disponible en: http://www.editoresmadrid.org/media/36706/seminario-1.pdf

Bernal Torres, C. (2006). Metodología de la investigación. Para administración, economía, humanidades y ciencias sociales. Segunda Edición. Person Educación. México.

Chiner, E. (2008). La validez. Universidada de Alicanta. https://rua.ua.es/dspace/bitstream/10045/19380/25/Tema\%206-Validez.pdf.

Constitución de la República del Ecuador (2008). Ciudad Alfaro: Asamblea Constituyente.

Dorronzoro, M. (2004). Construcción del proyecto lector en el contexto universitario. Simposio Leer y Escribir en la Educación Superior, I Congreso Internacional Educación, Lenguaje y Sociedad "Tensiones educativas en América Latina". Universidad Nacional de la Pampa, Santa Rosa, La Pampa, 1-3 de julio.

Dubs de Moya, R. (2002). El Proyecto Factible: una modalidad de investigación. Sapiens. Revista Universitaria de Investigación, vol. 3, núm. 2, diciembre, 2002, p. 0 Universidad Pedagógica Experimental Libertador. Caracas, Venezuela. Disponible en: http://www.redalyc.org/pdf/410/41030203.pdf

Fidias, G. (2012). El proyecto de Investigación. Editorial Episteme.

Gispert, M. (2000). Lectura y Memorización. Tomo 8. Barcelona: Océano.

Hernandez, A. (2014). El Proyecto Factible como Modalidad en la Investigación Educativa. Universidad Pedagógica Experimental Libertador (UPEL). Táchira. Venezuela

Hernández, R. Fernández, C. y Baptista, P. (2003). Metodología de la Investigación. México: Editorial McGraw Hill.

Hurtado, J. (2000). Metodología de la Investigación Holística. Sypal Caracas. Venezuela.

Pérez, M. (1999). La Lectura en la educación. Universidad Valle del Momboy. Trujillo, Venezuela.

Plan Nacional Para el Buen Vivir (2013-2017). Consejo Nacional de Planificación. República del Ecuador. 
Prieto, G. y Delgado, A. (2010). Fiabilidad y Validez. Papeles del Psicólogo, vol. 31, núm. 1, enero-abril, 2010, pp. 67-74. Consejo General de Colegios Oficiales de Psicólogos Madrid, España. Disponible en: http://www.redalyc.org/pdf/778/77812441007.pdf

Ynclán, G. (1997). Una historia sin fin. Crear y recrear texto. Fundación SNTE: México. 


\section{ANEXO A}

Estructura de la propuesta

Tabla No. 1. Actividades Dirigidas

$\begin{array}{ll}\text { Docentes } & \text { Estudiantes } \\ \Rightarrow \text { Determinar el propósito de la lectura. } & \Rightarrow \text { Identificar el propósito de la lectura. } \\ \Rightarrow \text { Indagar sobre saberes previos. } & \Rightarrow \text { Demostrar lo que conocen del tema. } \\ \Rightarrow \text { Desarrollar lecturas de texto. } & \Rightarrow \text { Leer textos de diversas formas. } \\ \begin{array}{ll}\Rightarrow \text { Utilizar la estrategia del resumen. } & \Rightarrow \text { Demostrar habilidades del uso del subrayado, } \\ \Rightarrow \text { Enfocar lecturas de artículos científicos. } & \text { organización de ideas y elaboración de resumen. } \\ \Rightarrow \text { Entregar material fotocopiado. } & \Rightarrow \text { Comentar libremente lo entendido en el material. } \\ \Rightarrow \text { Promover el uso de textos narrativos. } & \Rightarrow \text { Relatar acontecimientos reales ó ficticios. } \\ \Rightarrow \text { Utilizar espacios andragógicos fuera del aula. } & \Rightarrow \text { Participar y generar espacios de discusión. } \\ \Rightarrow \text { Diseñar y estructurar una biblioteca virtual. } & \Rightarrow \text { Concebir dos horas semanales para leer en forma } \\ \Rightarrow \text { Desarrollar concursos de producción de lectura. } & \Rightarrow \text { individual. } \\ \Rightarrow \text { Realizar talleres con la comunidad y estudiantes } \\ \begin{array}{l}\text { para discutir sobre la importancia de la lectura. } \\ \Rightarrow \text { Proyectar la animación como una estrategia } \\ \text { didáctica. }\end{array}\end{array} & \Rightarrow \text { Incentivar a la comunidad a participar. } \\ & \Rightarrow \text { Utilizar la tecnología para fortalecer la lectura. }\end{array}$

Mendoza (2017)

Tabla No. 2. Cuadro de estrategias didácticas para fortalecer el proceso de la lectura

\begin{tabular}{|c|c|c|}
\hline Objetivo General & Estrategias Didácticas & Evaluación \\
\hline $\begin{array}{l}\text { Implementar estrategias } \\
\text { didácticas que conduzcan a la } \\
\text { incentivación investigativa de } \\
\text { los estudiantes en La } \\
\text { Universidad Iberoamericana } \\
\text { del Ecuador, ubicada en Quito } \\
\text { República del Ecuador. }\end{array}$ & $\begin{array}{l}\text { - Exposición. } \\
\text { - Discusión grupal. } \\
\text { - Organizar situaciones para indagar los } \\
\text { saberes previos. } \\
\text { - Lecturas dirigidas. } \\
\text { - Resumen. } \\
\text { - Métodos de proyectos. } \\
\text { - Métodos de casos. } \\
\text { - Intercambio de preguntas. } \\
\text { - Simulación de juegos. } \\
\text { - Aprendizajes basados en problemas. } \\
\text { - Juegos de roles. } \\
\text { - Lluvias de ideas. }\end{array}$ & $\begin{array}{l}\text { - Lista de cotejo } \\
\text { - Observación } \\
\text { - Participación } \\
\text { - Intercambio de } \\
\text { experiencia. } \\
\text { - } \text { Mapas conceptuales } \\
\text { - Concursos de lectura }\end{array}$ \\
\hline
\end{tabular}

Mendoza (2017)

Desarrollo de las estrategias didácticas para fortalecer el proceso de la lectura 
Tabla No. 3. Discusión Grupal

\begin{tabular}{llllll}
\hline $\begin{array}{l}\text { Objetivo } \\
\text { Específico }\end{array}$ & Situación de Aprendizaje & Contenidos & $\begin{array}{l}\text { Recursos } \\
\text { Materiales }\end{array}$ & Tiempo & Evaluación \\
\cline { 2 - 5 } & Aula & & Textos y & 16 horas & Participación y \\
Fomentar & Se desarrollaran con los & ¿Qué es un libro? & Tluidez en la \\
habilidades & estudiantes(as) los & ¿Qué es un texto? & material & & lectura \\
sobre la & diferentes tipos de & Clasificación de & fotocopiado & & \\
lectura a & lectura y enfoques, a fin & los textos & & \\
través de la & de desarrollar & & & \\
discusión & habilidades que le & & & \\
grupal. & permitan realizar & & & \\
& lecturas críticas, & & & \\
& analíticas y & & & \\
& comprensivas. & & & \\
\end{tabular}

Mendoza (2017)

Tabla No. 4. Organizar situaciones para indagar los saberes previos

\begin{tabular}{|c|c|c|c|c|c|}
\hline \multirow{2}{*}{$\begin{array}{l}\text { Objetivo } \\
\text { Específico }\end{array}$} & \multicolumn{5}{|c|}{ Situación de Aprendizaje } \\
\hline & Aula & Contenidos & $\begin{array}{l}\text { Recursos } \\
\text { Materiales }\end{array}$ & Tiempo & Evaluación \\
\hline $\begin{array}{l}\text { Indagar sobre la } \\
\text { estructura } \\
\text { cognitiva que } \\
\text { tienen los } \\
\text { estudiantes(as) } \\
\text { sobre la lectura. }\end{array}$ & $\begin{array}{l}\text { Realizar un } \\
\text { intercambio de ideas a } \\
\text { fin de elaborar un } \\
\text { diagnóstico sobre el } \\
\text { dominio que tienen } \\
\text { los estudiantes(as) de } \\
\text { la lectura. Para ello, se } \\
\text { facilitará recortes de } \\
\text { prensa para que } \\
\text { demuestren } \\
\text { prácticamente sus } \\
\text { habilidades. }\end{array}$ & $\begin{array}{l}\text { Técnica de } \\
\text { indagación, } \\
\text { saberes previos. }\end{array}$ & $\begin{array}{l}\text { Periódicos, } \\
\text { revistas, folletos, } \\
\text { artículos } \\
\text { investigativos. }\end{array}$ & 16 horas & $\begin{array}{l}\text { Participación y } \\
\text { dominio en la } \\
\text { lectura. }\end{array}$ \\
\hline
\end{tabular}

Mendoza (2017)

Tabla No. 5. Lectura Dirigida

\begin{tabular}{|c|c|c|c|c|c|}
\hline \multirow{2}{*}{$\begin{array}{l}\text { Objetivo } \\
\text { Específico }\end{array}$} & \multicolumn{5}{|l|}{ Situación de Aprendizaje } \\
\hline & Aula & Contenidos & $\begin{array}{l}\text { Recursos } \\
\text { Materiales }\end{array}$ & Tiempo & Evaluación \\
\hline $\begin{array}{l}\text { Orientar la } \\
\text { lectura de } \\
\text { manera que } \\
\text { sea productiva } \\
\text { y eficaz para } \\
\text { los } \\
\text { estudiantes. }\end{array}$ & $\begin{array}{l}\text { Desarrollar lecturas en } \\
\text { voz alta, para lo cual el } \\
\text { docente debe participar } \\
\text { en cuales son los } \\
\text { objetivos que debe } \\
\text { trabajar y además } \\
\text { permitirles a los } \\
\text { estudiantes(as) que } \\
\text { elijan los textos de su } \\
\text { interés, a fin que se } \\
\text { disfrute de ello. De igual } \\
\text { manera, la búsqueda de } \\
\text { la comprensión y } \\
\text { ampliar el vocabulario, } \\
\text { buscar la atención, }\end{array}$ & $\begin{array}{l}\text { Lectura de } \\
\text { calidad científica. }\end{array}$ & $\begin{array}{l}\text { Revistas, } \\
\text { folletos, } \\
\text { artículos } \\
\text { investigativos, } \\
\text { libros. }\end{array}$ & 16 horas & $\begin{array}{l}\text { Ilustraciones, } \\
\text { diagramas y } \\
\text { esquemas. }\end{array}$ \\
\hline
\end{tabular}


imaginación y la

memoria

Mendoza (2017)

Tabla No. 6. Resumen

\begin{tabular}{|c|c|c|c|c|c|}
\hline \multirow{2}{*}{$\begin{array}{l}\text { Objetivo } \\
\text { Específico }\end{array}$} & \multicolumn{5}{|l|}{ Situación de Aprendizaje } \\
\hline & Aula & Contenidos & $\begin{array}{l}\text { Recursos } \\
\text { Materiales }\end{array}$ & Tiempo & Evaluación \\
\hline $\begin{array}{l}\text { Desarrollar } \\
\text { competencias } \\
\text { necesarias } \\
\text { para que los } \\
\text { estudiantes } \\
\text { identifiquen } \\
\text { las ideas } \\
\text { principales de } \\
\text { un texto. }\end{array}$ & $\begin{array}{l}\text { Explicar a los } \\
\text { estudiantes las reglas } \\
\text { que se deben utilizar } \\
\text { para recibir el contenido } \\
\text { de un texto como son: } \\
\text { omitir, seleccionar, } \\
\text { generalizar, construir ó } \\
\text { integrar. Asimismo, que } \\
\text { los alumnos } \\
\text { comprendan el por qué } \\
\text { se necesita resumir, } \\
\text { también conocer, que } \\
\text { para hacer un resumen } \\
\text { hay que leer el texto, } \\
\text { subrayar la idea } \\
\text { principal de cada } \\
\text { párrafo, realizar un } \\
\text { esquema, explicar } \\
\text { claramente la esencia } \\
\text { del texto, no usar } \\
\text { exactamente las palabras } \\
\text { del original y recordar } \\
\text { que el resumen es } \\
\text { distinto al comentario, } \\
\text { por lo tanto, no hace } \\
\text { falta que escriba sus } \\
\text { opiniones. }\end{array}$ & $\begin{array}{l}\text { El resumen, } \\
\text { características, } \\
\text { reglas. }\end{array}$ & $\begin{array}{l}\text { Libros, } \\
\text { Revistas, } \\
\text { folletos, } \\
\text { artículos } \\
\text { investigativos, } \\
\text { periódicos, } \\
\text { material } \\
\text { fotocopiado. }\end{array}$ & 16 horas & $\begin{array}{l}\text { Habilidad en el } \\
\text { uso del } \\
\text { subrayado, } \\
\text { organización de } \\
\text { las ideas, } \\
\text { exactitud del } \\
\text { resumen. }\end{array}$ \\
\hline
\end{tabular}

Mendoza (2017)

Tabla No. 7. Motivar a los estudiantes al proceso de lectura

\begin{tabular}{|c|c|c|c|c|c|}
\hline \multirow{2}{*}{$\begin{array}{l}\text { Objetivo } \\
\text { Específico }\end{array}$} & \multicolumn{5}{|l|}{ Situación de Aprendizaje } \\
\hline & Aula & Contenidos & $\begin{array}{l}\text { Recursos } \\
\text { Materiales }\end{array}$ & Tiempo & Evaluación \\
\hline $\begin{array}{l}\text { Elaboración de } \\
\text { manuscritos de } \\
\text { investigación } \\
\text { académica } \\
\text { para } \\
\text { fortalecimiento } \\
\text { de la lectura } \\
\text { en los } \\
\text { estudiantes. }\end{array}$ & $\begin{array}{l}\text { Explicar a los } \\
\text { estudiantes(as) sobre la } \\
\text { redacción de artículos } \\
\text { investigativos, sus } \\
\text { partes, tipos de estudios, } \\
\text { estructuraciones, a fin } \\
\text { de incentivar a los } \\
\text { estudiantes(as) para el } \\
\text { desarrollo de este } \\
\text { ratificando la ayuda que } \\
\text { fomenta al hábito de la } \\
\text { lectura y viene a } \\
\text { constituirse como una } \\
\text { estrategia de apoyo en }\end{array}$ & $\begin{array}{l}\text { Periódico mural, } \\
\text { características, } \\
\text { tipo de } \\
\text { información, } \\
\text { conformación de } \\
\text { las sesiones, tipo } \\
\text { de papel, } \\
\text { ilustraciones. }\end{array}$ & $\begin{array}{l}\text { Artículos } \\
\text { científicos de } \\
\text { investigación, } \\
\text { Libros, } \\
\text { Revistas, } \\
\text { folletos, } \\
\text { material } \\
\text { fotocopiado. }\end{array}$ & 16 horas & $\begin{array}{l}\text { Participación, } \\
\text { observación, } \\
\text { redacción } \\
\text { científica, } \\
\text { registros. }\end{array}$ \\
\hline
\end{tabular}


las diferentes áreas

académicas. Asimismo,

esta estrategia es valiosa

porque el alumno está

en contacto visual

constante con la

información y logrará

mayor retención de lo

aprendido.

Mendoza (2017)

Tabla No. 8. Diario de vida

\begin{tabular}{|c|c|c|c|c|c|}
\hline \multirow{2}{*}{$\begin{array}{l}\text { Objetivo } \\
\text { Específico }\end{array}$} & \multicolumn{5}{|l|}{ Situación de Aprendizaje } \\
\hline & Aula & Contenidos & $\begin{array}{l}\text { Recursos } \\
\text { Materiales }\end{array}$ & Tiempo & Evaluación \\
\hline $\begin{array}{l}\text { Incentivar a } \\
\text { los estudiantes } \\
\text { a narrar } \\
\text { hechos para el } \\
\text { fortalecimiento } \\
\text { de la lectura y } \\
\text { la } \\
\text { comunicación. }\end{array}$ & $\begin{array}{l}\text { Se les indicara a los } \\
\text { estudiantes sobre la } \\
\text { elaboración de un diario } \\
\text { de vida, en el cual } \\
\text { deben ir realizando } \\
\text { anotaciones hechas cada } \\
\text { semana en donde } \\
\text { escriban los sucesos } \\
\text { más importantes y las } \\
\text { experiencias más } \\
\text { significativas. Luego, } \\
\text { mensualmente el } \\
\text { estudiante debe } \\
\text { compartir sus } \\
\text { manuscritos a través de } \\
\text { la lectura con sus } \\
\text { compañeros de clases, a } \\
\text { su vez servirá para } \\
\text { fortalecer habilidades } \\
\text { en la escritura y } \\
\text { redacción. }\end{array}$ & $\begin{array}{l}\text { Formas verbales, } \\
\text { el tema, } \\
\text { argumento, } \\
\text { estructura, } \\
\text { tiempo, } \\
\text { personaje y } \\
\text { espacio. }\end{array}$ & $\begin{array}{l}\text { Papel, lápiz, } \\
\text { esferos, } \\
\text { tarjetas, } \\
\text { colores, } \\
\text { marcadores. }\end{array}$ & $\begin{array}{l}\text { Durante } \\
\text { todo el } \\
\text { semestre } \\
\text { académico. }\end{array}$ & $\begin{array}{l}\text { Participación, } \\
\text { creatividad, } \\
\text { imaginación, } \\
\text { redacción, } \\
\text { registros. }\end{array}$ \\
\hline
\end{tabular}

Mendoza (2017)

Tabla No. 9. Aprender Jugando

\begin{tabular}{|c|c|c|c|c|c|}
\hline \multirow{2}{*}{$\begin{array}{l}\text { Objetivo } \\
\text { Específico }\end{array}$} & \multicolumn{5}{|l|}{ Situación de Aprendizaje } \\
\hline & Aula & Contenidos & $\begin{array}{l}\text { Recursos } \\
\text { Materiales }\end{array}$ & Tiempo & Evaluación \\
\hline $\begin{array}{l}\text { Fomentar la } \\
\text { lectura a } \\
\text { partir de las } \\
\text { actividades } \\
\text { lúdicas. }\end{array}$ & $\begin{array}{l}\text { Motivar a los } \\
\text { estudiantes para } \\
\text { participar en el juego } \\
\text { poético, para ello, se } \\
\text { debe crear previamente } \\
\text { un clima propicio a una } \\
\text { audición, se reparten } \\
\text { copias de los poemas } \\
\text { para la lectura } \\
\text { individual al mismo } \\
\text { tiempo, se eligen varios } \\
\text { poemas, que se someten } \\
\text { a la selección de la }\end{array}$ & $\begin{array}{l}\text { El poema, } \\
\text { características, } \\
\text { tipos de poemas. }\end{array}$ & $\begin{array}{l}\text { Papel, lápiz, } \\
\text { esferos, } \\
\text { tarjetas, } \\
\text { colores, } \\
\text { marcadores. }\end{array}$ & $\begin{array}{l}\text { Durante } \\
\text { todo el } \\
\text { semestre } \\
\text { académico. }\end{array}$ & $\begin{array}{l}\text { Participación, } \\
\text { creatividad, } \\
\text { imaginación, } \\
\text { redacción, } \\
\text { registros. }\end{array}$ \\
\hline
\end{tabular}


mayoría de los

estudiantes, luego se da

lectura al poema

cuidando espacialmente

la entonación y ritmo,

luego se incentiva al

estudiante a representar

a través del dibujo el

poema descrito.

Mendoza (2017)

Tabla No. 10. Crear una biblioteca virtual

\begin{tabular}{|c|c|c|c|c|c|}
\hline \multirow{2}{*}{$\begin{array}{l}\text { Objetivo } \\
\text { Específico }\end{array}$} & \multicolumn{5}{|c|}{ Situación de Aprendizaje } \\
\hline & Aula & Contenidos & $\begin{array}{l}\text { Recursos } \\
\text { Materiales }\end{array}$ & Tiempo & Evaluación \\
\hline $\begin{array}{l}\text { Incentivar a los } \\
\text { estudiantes } \\
\text { para la } \\
\text { construcción de } \\
\text { un espacio } \\
\text { virtual de } \\
\text { lectura } \\
\text { mediantes las } \\
\text { Tecnologías de } \\
\text { Información y } \\
\text { Comunicación } \\
\text { (TIC). }\end{array}$ & $\begin{array}{l}\text { Ubicar el rincón de } \\
\text { lectura digital, para } \\
\text { ello, se contará con } \\
\text { el apoyo del } \\
\text { personal que labora } \\
\text { el laboratorio de } \\
\text { computación, } \\
\text { trabajar en conjunto } \\
\text { con los } \\
\text { estudiantes(as) para } \\
\text { la recolección de } \\
\text { libros digitalizados } \\
\text { en formato de } \\
\text { documento portátil } \\
\text { (PDF), planificar } \\
\text { con ellos el horario } \\
\text { respectivo y para } \\
\text { los círculos de } \\
\text { lectura, clasificar el } \\
\text { material de lectura, } \\
\text { estimular a los } \\
\text { estudiantes(as) para } \\
\text { que lleven un } \\
\text { registro del material } \\
\text { leído y facilitar una } \\
\text { carpeta donde ellos } \\
\text { opinen y den } \\
\text { recomendaciones } \\
\text { sobre los libros que } \\
\text { van leyendo, } \\
\text { asignar para cada } \\
\text { semana un } \\
\text { responsable en la } \\
\text { entrega y } \\
\text { recolección de } \\
\text { material de lectura. }\end{array}$ & $\begin{array}{l}\text { Importancia del } \\
\text { rincón de } \\
\text { lectura digital, } \\
\text { características y } \\
\text { formas para } \\
\text { seleccionar el } \\
\text { material de } \\
\text { lectura, normas } \\
\text { para mantener la } \\
\text { plataforma } \\
\text { digital } \\
\text { interactiva de } \\
\text { lectura. }\end{array}$ & $\begin{array}{l}\text { Red Internet, } \\
\text { equipo } \\
\text { tecnológico con } \\
\text { sistema operativos } \\
\text { Android (versión } \\
4.0 \text { o superior), } \\
\text { Computador con } \\
\text { sistema operativo } \\
\text { Windows (versión } \\
\text { XP, Vista, } 7,8 \text { o } \\
\text { 10), Papel, lápiz, } \\
\text { libros, revistas, } \\
\text { periódicos, } \\
\text { folletos, artículos } \\
\text { científicos, entre } \\
\text { otros. }\end{array}$ & $\begin{array}{l}\text { Durante } \\
\text { todo el } \\
\text { semestre } \\
\text { académico. }\end{array}$ & $\begin{array}{l}\text { Participación, } \\
\text { lista de cotejo, } \\
\text { pertinencia. }\end{array}$ \\
\hline
\end{tabular}

Mendoza (2017) 
Tabla No. 11. Talleres de Lectura

\begin{tabular}{|c|c|c|c|c|c|}
\hline \multirow{2}{*}{$\begin{array}{l}\text { Objetivo } \\
\text { Específico }\end{array}$} & \multicolumn{5}{|c|}{ Situación de Aprendizaje } \\
\hline & Aula & Contenidos & $\begin{array}{l}\text { Recursos } \\
\text { Materiales }\end{array}$ & Tiempo & Evaluación \\
\hline $\begin{array}{l}\text { Incentivar a } \\
\text { los familiares, } \\
\text { amistades y } \\
\text { residentes de } \\
\text { la comunidad } \\
\text { a desarrollar } \\
\text { procesos que } \\
\text { fortalezcan la } \\
\text { lectura en el } \\
\text { estudiantado. }\end{array}$ & $\begin{array}{l}\text { Discutir con la } \\
\text { sociedad la } \\
\text { importancia de crear } \\
\text { hábitos de lectura. } \\
\text { Unificar criterios para } \\
\text { incentivar al educando } \\
\text { por el proceso lector, } \\
\text { organizar diferentes } \\
\text { espacios para que } \\
\text { disfruten de la lectura, } \\
\text { aplicación de } \\
\text { estrategias de } \\
\text { activación, } \\
\text { procesamiento de } \\
\text { información, } \\
\text { recapitulación de lo } \\
\text { que se pretende lograr. }\end{array}$ & $\begin{array}{l}\text { Importancia de } \\
\text { la lectura, } \\
\text { necesidad de } \\
\text { fortalecer los } \\
\text { procesos de la } \\
\text { lectura, redes } \\
\text { comunitarias. }\end{array}$ & $\begin{array}{l}\text { Papel, lápiz, } \\
\text { esfero, } \\
\text { cartulina, } \\
\text { material } \\
\text { fotocopiado, } \\
\text { papel bond, } \\
\text { proyector } \\
\text { audiovisual. }\end{array}$ & 1 hora & $\begin{array}{l}\text { Participación, } \\
\text { pertinencia, } \\
\text { autoevaluación, } \\
\text { conclusiones. }\end{array}$ \\
\hline
\end{tabular}

